\title{
Low-temperature localization in the transport properties of self-doped $\mathrm{La}_{0.9} \mathrm{Mn}_{0.98} \mathrm{Zn}_{0.02} \mathrm{O}_{3}$
}

\author{
$\mathrm{K} \mathrm{DE}^{1}$ and $\mathrm{S} \mathrm{DAS}^{2, *}$ \\ ${ }^{1}$ Neotia Institute of Technology, Management and Science, Jhinga 743 368, India \\ ${ }^{2}$ Department of Electronics and Communication Engineering, Guru Ghasidas Central University, \\ Bilaspur (C.G.) 495 009, India
}

MS received 9 August 2015; accepted 1 October 2015

\begin{abstract}
Low-temperature transport properties are investigated in the self-doped compound, $\mathrm{La}_{0.9} \mathrm{Mn}_{0.98}$ $\mathrm{Zn}_{0.02} \mathrm{O}_{3}$. The analysis of the low-temperature resistivity is performed considering various scattering mechanisms. The parameters involved with different scattering processes such as electron-electron, Kondo, electron-phonon and electron-magnon are found to be strongly influenced by the applied magnetic field. The results suggest that interplay between electron-electron and Kondo-like scatterings lead to the localization in the temperature dependence of resistivity at low temperature.
\end{abstract}

Keywords. Transport properties; scattering mechanisms; low temperature localization.

\section{Introduction}

The investigation on mixed-valent manganites with perovskite structure is on run for the last two decades. Specially, the studies on the hole doped manganites, $\mathrm{La}_{1-\delta} \mathrm{A}_{\delta} \mathrm{MnO}_{3}$ $(\mathrm{A}=$ divalent atom $)$ still demands special attention because of the intriguing magnetic and transport properties [1-5]. In recent times, the self-doped manganites with composition $\mathrm{La}_{1-\delta} \mathrm{MnO}_{\mathrm{Q}}$ also display analogous features in the magnetic, transport and structural properties depending on $\mathrm{Q}$ and $\delta$ [6-15]. $\mathrm{Mn}^{4+}$ appears in these compounds due to the La deficiency, leading to the spectacular changes in the physical properties.

In recent times, the electronic transport in few manganites displays localization effect in the metallic region at low temperature, suggesting different opinions [16-23]. The low-temperature localization in resistivity was suggested due to the grain boundary effect in $\mathrm{La}_{0.5} \mathrm{~Pb}_{0.5} \mathrm{MnO}_{3}$ [16]. The electron-electron (e-e) scattering ascribed to the enhanced Coulomb interaction was interpreted in $\mathrm{La}_{0.8} \mathrm{Sr}_{0.2} \mathrm{MnO}_{3}$ and thin film of $\mathrm{La}_{0.7} \mathrm{Ca}_{0.3} \mathrm{MnO}_{3}$ [17,18]. The coexistence of Kondo-like behaviour and e-e scattering has been proposed in the polycrystalline hole doped manganites, $\mathrm{La}_{2 / 3} \mathrm{Ca}_{1 / 3} \mathrm{MnO}_{3}$ and Y-doped $\left(\mathrm{La}_{1-\delta} \mathrm{Y}_{\delta}\right)_{2 / 3} \mathrm{Ca}_{1 / 3} \mathrm{MnO}_{3}$ $[20,21]$. The increase of localization effect as a result of minor $\mathrm{Co}$ and $\mathrm{Fe}$ substitutions has also been investigated in $\mathrm{La}_{0.87} \mathrm{Mn}_{0.98} \mathrm{M}_{0.02} \mathrm{O}_{3}(\mathrm{M}=\mathrm{Fe}$ and $\mathrm{Co})$ where minor magnetic impurity or spin-glass-like phase appeared due to the substitution resulted in the dominant localization effect $[22,23]$. In contrast to the substitution by magnetic impurity such as $\mathrm{Fe}$ and $\mathrm{Co}$, the substitution of nonmagnetic $\mathrm{Zn}$

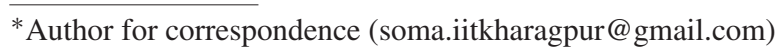

also decreases the conductivity and lowering of Curie temperature in $\mathrm{La}_{0.91} \mathrm{Mn}_{0.95} \mathrm{Zn}_{0.05} \mathrm{O}_{3}$ [24]. Signature of glassy magnetic behaviour and decrease of conductivity ascribed to the disorder created by the $\mathrm{Zn}$ substitution was observed in $\mathrm{LaMn}_{1-x} \mathrm{Zn}_{x} \mathrm{O}_{3}$ [25]. The minor $\mathrm{Zn}$ substitution in hole doped, $\mathrm{La}(\mathrm{Ca}) \mathrm{MnO}_{3}$ modifies the grain boundary, resulting in the decrease of conductivity [26-28].

In this article, the low-temperature localization effect due to the minor $\mathrm{Zn}$ substitution in $\mathrm{La}_{0.9} \mathrm{Mn}_{0.98} \mathrm{Zn}_{0.02} \mathrm{O}_{3}$ is investigated. At low temperature the localization effect is confirmed due to the interplay between e-e and Kondo scatterings in accordance with the reported phenomenological models.

\section{Experimental}

The polycrystalline compound with composition $\mathrm{La}_{0.9} \mathrm{Mn}_{0.98}$ $\mathrm{Zn}_{0.02} \mathrm{O}_{3}$ was prepared by the chemical route as described in our earlier literature [29]. The final annealing was performed at $1200^{\circ} \mathrm{C}$ for $12 \mathrm{~h}$ in air followed by furnace cooling down to room temperature. In order to avoid ambiguity ascribed to the oxygen non-stoichiometry, sample was again annealed in atmospheric pressure of oxygen for $6 \mathrm{~h}$ at $1000^{\circ} \mathrm{C}$. The single phase of the sample was confirmed by the powder X-ray diffraction (XRD) (Seiferd XRD 3000P) using $\mathrm{CuK}_{\alpha}$ radiation. All the diffraction peaks could be indexed in the rhombohedral structure $(\mathrm{R} \overline{3} \mathrm{c})$ with lattice parameters $a=5.47(2) \AA$ and $\alpha=60.59^{\circ}$. Scanning electron microscopy (SEM) image in figure 1 shows the size and morphology of the grains in the sample. The particles exist as big grains with not well-defined edges. The inhomogeneity of the grain size with average diameter $\approx 90 \mathrm{~nm}$ was noticed 


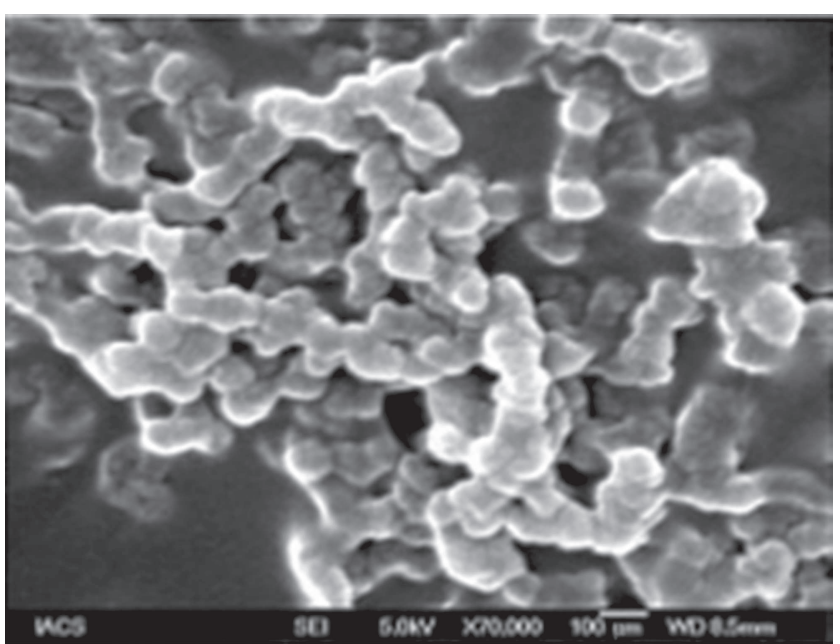

Figure 1. SEM image for $\mathrm{La}_{0.9} \mathrm{Mn}_{0.98} \mathrm{Zn}_{0.02} \mathrm{O}_{3}$.

in the SEM observation. The resistivity $(\rho)$ was measured using the standard four-probe technique where application of the magnetic field and temperature variation were carried out by an electromagnet (Polytronic Corp., India) and a cryogenfree cryostat operating down to $10 \mathrm{~K}$ (Janis Research Inc., USA), respectively. Magneto-resistance (MR) is defined as $[\{\rho(H)-\rho(H=0)\} / \rho(H=0)]$ where $\rho(H)$ is the resistivity measured in field $(H)$. All the thermal variation during resistivity measurement was carried out at a fixed rate of $2 \mathrm{~K} \mathrm{~min}^{-1}$. The magnetization (M) was measured using a commercial superconducting quantum interference device (SQUID) magnetometer (MPMS, XL). In case of zero-field cooled (ZFC) mode the sample was cooled down to the desired temperature at zero magnetic field while for the fieldcooled (FC) mode the sample was cooled in a static magnetic field.

\section{Results and discussions}

Temperature variation of resistivity $\rho$ measured in zero-field and $2 \mathrm{kOe}$ field is shown in figure 2 for $\mathrm{La}_{0.9} \mathrm{Mn}_{0.98} \mathrm{Zn}_{0.02} \mathrm{O}_{3}$. A sharp peak in resistivity $\rho$ is observed at $235 \mathrm{~K}\left(T_{\mathrm{P}}\right)$ which is accompanied by a broad maximum around $\sim 175 \mathrm{~K}\left(T_{\mathrm{m}}\right)$. The features in the temperature dependence of resistivity $\rho$ are in accordance with the parent compound, $\mathrm{La}_{0.9} \mathrm{MnO}_{3}$ where $T_{\mathrm{P}}$ and $T_{\mathrm{m}}$ are shifted towards the low temperature in the present observation. The result is also consistent with those reported in $\mathrm{La}_{0.9} \mathrm{Mn}_{1-x} \mathrm{Fe}_{x} \mathrm{O}_{3}(0 \leq x \leq 0.05)$ [22] and $\mathrm{La}_{0.9} \mathrm{Mn}_{0.98} \mathrm{M}_{0.02} \mathrm{O}_{3}(M=\mathrm{O}, \mathrm{Fe}$ and $\mathrm{Co})$ [23]. It was noted that the considerable increase of $\rho$ involved with the minimal $\mathrm{Zn}$ substitution in the entire temperature range compared to the parent compound. This is in accordance with the minor $\mathrm{Zn}$ substitution in $\mathrm{LaMnO}_{3}$ as well as hole doped, $\mathrm{La}(\mathrm{Ca}) \mathrm{MnO}_{3}$ where disorder introduced by the $\mathrm{Zn}$ substitution, partial disruption of double-exchange interaction, modification of the grain boundary effect have been proposed to interpret the results [25-27].

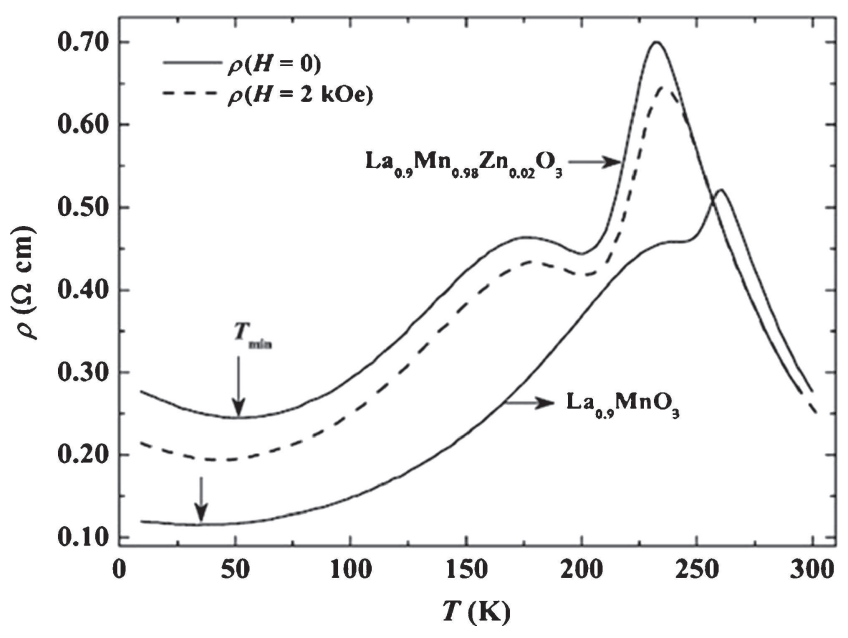

Figure 2. Temperature dependence resistivity $(\rho)$ in zero field for compounds $\mathrm{La}_{0.9} \mathrm{Mn}_{0.98} \mathrm{Zn}_{0.02} \mathrm{O}_{3}$ and $\mathrm{La}_{0.9} \mathrm{MnO}_{3}$.

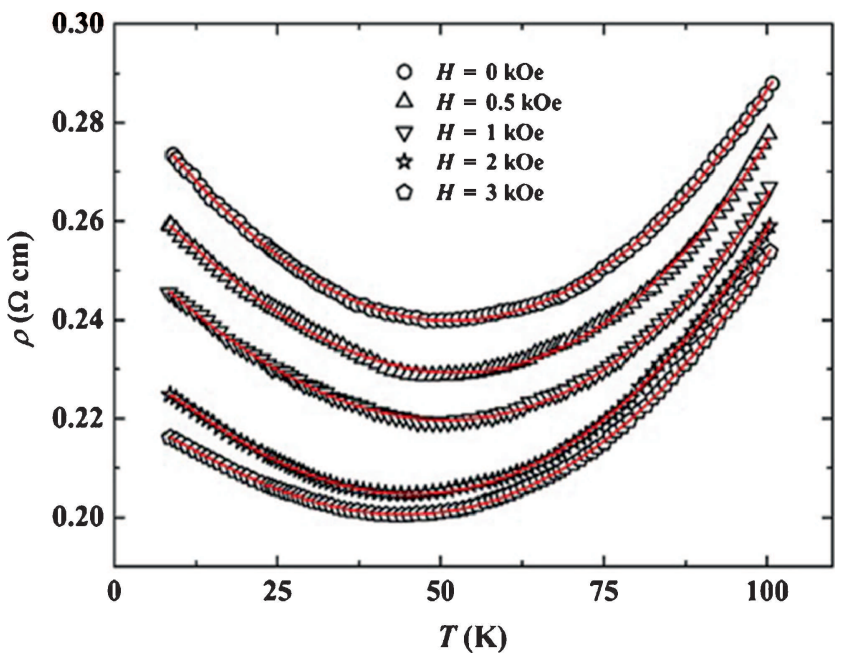

Figure 3. Temperature dependence resistivity $(\rho)$ under ZFC condition with different applied fields, the solid lines indicate the fitted results by using equation (3).

Temperature dependence of $\rho$ by varying field, $H$ ranging from 0 to $3.0 \mathrm{kOe}$ is shown in figure 3 below $100 \mathrm{~K}$. It has been noted that resistivity minimum appeared at $T_{\min }$ is shifted towards high temperature compared to the parent compound [23]. Furthermore, $T_{\min }$ shows the strong field dependence where $T_{\min }$ is shifted towards low temperature with the increase field which is shown in the inset of figure 4 . In figure 4 the resistivity upturn, $\rho_{10 \mathrm{~K}}$ defined as $\left\{\rho_{10 \mathrm{~K}}-\right.$ $\left.\rho\left(T_{\min }\right)\right\} / \rho\left(T_{\min }\right)(\%)$ is plotted as a function of $H$ where $\rho_{10 \mathrm{~K}}$ and $\rho\left(T_{\min }\right)$ are the resistivities at $10 \mathrm{~K}$ and minimum in the temperature dependence of $\rho$, respectively. The plot clearly demonstrates that low- $T$ resistivity is strongly influenced by the magnetic field. Therefore, it can be suggested that the spin-dependent scattering must be associated with the resistivity minima of this sample which is gradually suppressed by external magnetic field. 


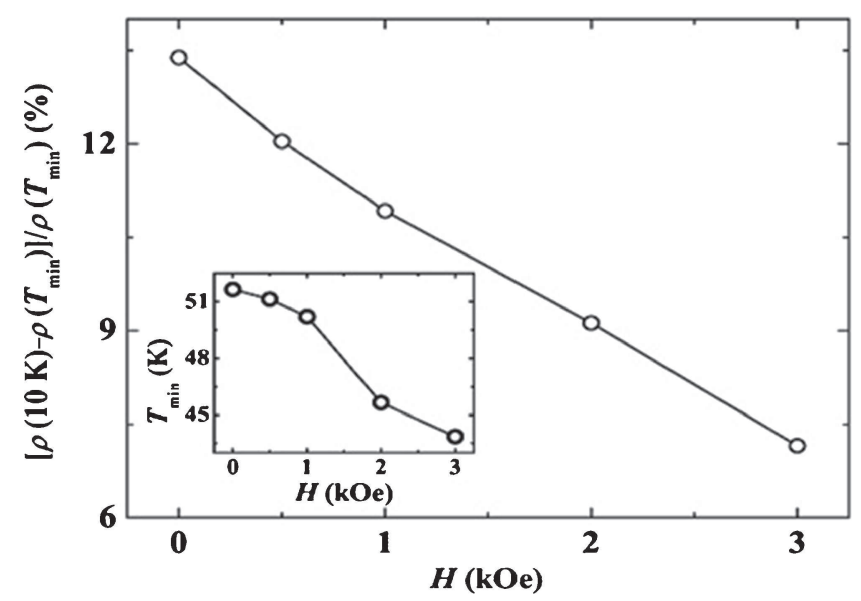

Figure 4. $\Delta \rho_{10 \mathrm{~K}}=\left(\rho_{10 \mathrm{~K}}-\rho\left(T_{\min }\right)\right) / \rho\left(T_{\min }\right)(\%)$ as a function of the applied field. The inset shows the dependence of $T_{\min }$ on the applied field, $H$.

In order to elucidate the origin of low- $T$ upturn different phenomenological models have been proposed considering spin-dependent Kondo-like scattering, e-e interaction, weak localization, inter-granular tunnelling of polarized charge carriers, etc [16-21]. The e-e scattering due to Coulomb interaction between the charge carriers has been proposed in references [30-32]. Note that interpretation using e-e may be adopted in the analysis when the magnitude of lowtemperature resistivity is higher than Motts maximum limit of metallic resistivity with $\rho \approx 10 \mathrm{~m} \Omega \mathrm{cm}[30,31]$, which for the present sample is well satisfied. In order to test the possible e-e interaction, $T^{1 / 2}$ dependence of $\rho(T)-\rho_{\min }$ at different $H$ is plotted in figure 5 for $T \leq T_{\min }$. The figure shows that the plots fit satisfactory over a wide temperature range below $T_{\min }$ at different fields. We also tried to fit all the curves by taking into account the Kondo scattering term $\ln (T)$ which has been used to fit the low- $T$ resistivity in few manganites $[18,20,21]$. The inset of figure 5 shows the $\ln (T)$ dependence of resistivity in zero field. The plot indicates that it fits satisfactorily in a limited lowtemperature region which departs at much lower temperature than $T_{\min }$ indicated by the arrows in the figure. In addition to the elastic e-e scattering and Kondo-like scattering, the resistivity in the metallic region is ascribed to the various inelastic contributions such as electron-phonon and electron-magnon scattering processes. At low temperature e-e and Kondo scatterings dominate while the inelastic term dominates at high temperature. Therefore, the resistivity may be defined by adding both the elastic and inelastic scattering terms as

$$
\rho=\rho_{\text {elastic }}+\rho_{\text {inelastic }} .
$$

Considering elastic e-e scattering, $\rho_{\text {elastic }}$ is given by $\rho_{\text {elastic }}=$ $1 /\left[\sigma(0)+B T^{1 / 2}\right]$ where $\sigma(0)$ is the residual conductivity and $B$ the constant $[30,31]$. The inelastic scattering term is given by a power law, $\rho_{\text {inelastic }}=A T^{n}$. This single power law adequately describes all the temperature-dependent inelastic

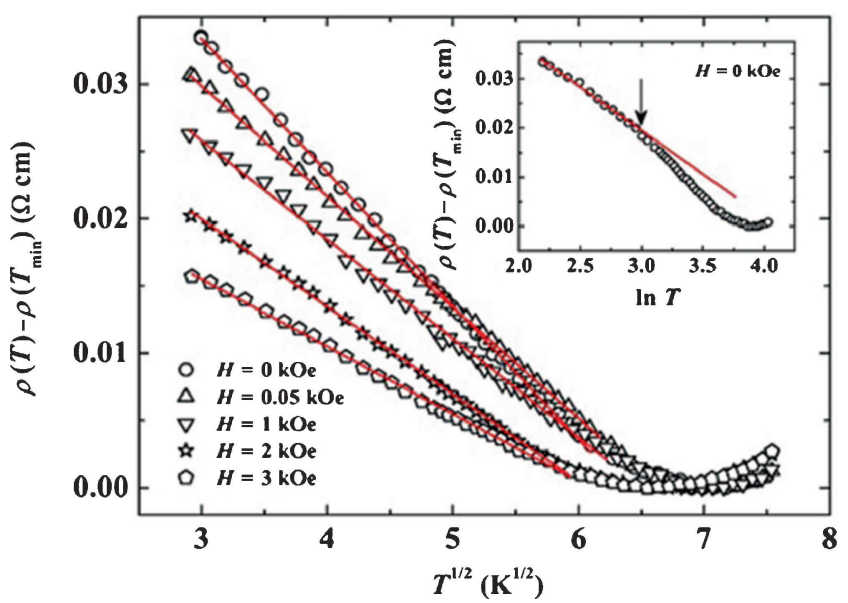

Figure 5. $T^{1 / 2}$ dependence of resistivity subtracted the minimum value defined as $\rho(T)-\rho\left(T_{\min }\right)$ under different applied fields $(H \leq$ $3 \mathrm{kOe}$ ). The solid lines are the fitting results. The inset shows the dependence of $\ln T$ with $\rho(T)-\rho\left(T_{\min }\right)$, where solid lines are indicated the linear fits.

scattering processes at high temperature. Thus, equation (1) is redefined as

$$
\rho(T)=\frac{1}{\sigma(0)+B T^{1 / 2}}+A T^{n} .
$$

The interplay between these two temperature-dependent terms in equation (2) leads to the resistivity minimum in the temperature dependence. At low field the grain boundary effect is also typically observed in the magneto-resistance for granular manganites having ferromagnetic ground state $[15,33]$. Therefore, as discussed before, in addition to the elastic e-e scattering, a Kondo scattering term $\ln (T)$ has been introduced for interpreting the low- $T$ upturn $[20,21]$ and it has been noted that the degree of fitting $\left(\chi^{2}\right)$ is improved further by one order of magnitude after considering this additional $\ln (T)$ term for the analysis of the experimental data. Thus, the following phenomenological relation $[20,21]$, where all the scattering terms are separated:

$$
\rho(T)=\rho_{0}+\rho_{\mathrm{e}} T^{1 / 2}-\rho_{\mathrm{s}} \ln T+\rho_{\mathrm{p}} T^{n}
$$

is used to fit the $\rho(T)$ data for all $H$ and is shown by the solid lines in figure 6 . The low values of $\chi^{2}\left(\sim 10^{-7}\right)$ give satisfactory fit with the experimental data. The strong magnetic field dependence is noticed in all the fitted parameters. Inelastic term involved with the electron-phonon and electronmagnon scattering is found to be several orders of magnitude smaller than the other contributions. Inelastic scattering exponent $(n)$ is found in between 2.39 and 2.75 (table 1) which is close to that observed in $\mathrm{La}_{1-\delta} \mathrm{Ca}_{\delta} \mathrm{MnO}_{3}$ by Schiffer et al [34] with $n=25$. Table 1 shows that the values of $\rho_{0}, \rho_{\mathrm{e}}$ and $\rho_{\mathrm{s}}$ decrease monotonically with applied magnetic analogous to that observed in $\mathrm{La}_{09} \mathrm{Mn}_{098} \mathrm{M}_{002} \mathrm{O}_{3}(\mathrm{M}=\mathrm{O}, \mathrm{Fe}$ and $\mathrm{Co}), \mathrm{La}_{2 / 3} \mathrm{Ca}_{1 / 3} \mathrm{MnO}_{3}$ and Y-doped $\left(\mathrm{La}_{1-\delta} \mathrm{Y}_{\delta}\right)_{2 / 3} \mathrm{Ca}_{1 / 3} \mathrm{MnO}_{3}$ $[20,21,23]$. The decrease of residual resistivity $\rho_{0}$ with the increase in $H$ is different from the usual conductor where 


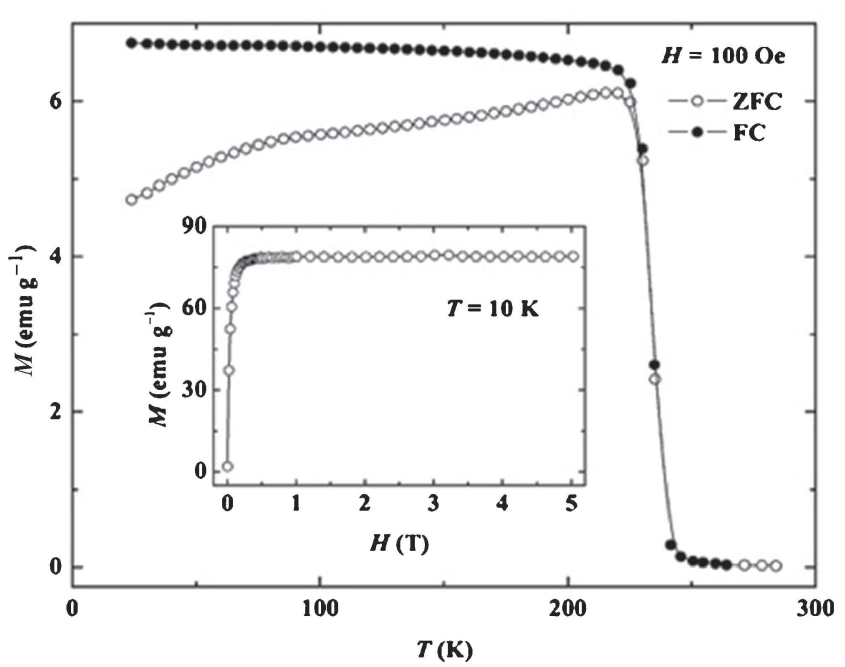

Figure 6. Temperature dependence of ZFC (open symbols) and FC (solid symbols) magnetizations in an applied field of 100 Oe. Inset shows the magnetization as a function of applied field at $10 \mathrm{~K}$.

Table 1. Parameters are obtained from the fits of $\rho-T$ curves in figure 3 to equation (3).

\begin{tabular}{lcccccc}
\hline$H(\mathrm{kOe})$ & $\rho_{0}$ & $\rho_{\mathrm{e}}$ & $\rho_{\mathrm{s}}$ & $\rho_{\mathrm{p}}\left(10^{-6}\right)$ & $n$ & $\chi^{2}\left(10^{-7}\right)$ \\
\hline 0 & 0.297 & 0.020 & 0.018 & 1.99 & 2.39 & 1.10 \\
0.5 & 0.276 & 0.018 & 0.016 & 0.42 & 2.69 & 3.60 \\
1.0 & 0.263 & 0.014 & 0.015 & 0.28 & 2.75 & 1.50 \\
2.0 & 0.238 & 0.012 & 0.013 & 1.43 & 2.45 & 0.77 \\
3.0 & 0.226 & 0.012 & 0.012 & 0.47 & 2.65 & 0.38 \\
\hline
\end{tabular}

$\rho_{0}$ generally is independent of $T$ and $H$. In case of manganites, $\rho_{0}$ may be related to intrinsic properties of system and reflects CMR effect and disorder characteristics of the system. From table $1, \rho_{\mathrm{p}}$ does not show monotonic change with $H$ and may be interpreted to be field insensitive and is smaller in few orders of magnitudes than the other effects at low temperatures. Compared to $\rho_{\mathrm{p}}$ (the inelastic scattering), the values of $\rho_{\mathrm{e}}$ and $\rho_{\mathrm{s}}$ are much higher, both are positive and comparable to each other at all fields. Both are monotonically decreasing with the increase in field. This explains the decrease of resistivity with field at low temperature due to suppression of spin-dependent scattering resulting in $\Delta \rho_{10 \mathrm{~K}}$ and shifting of $T_{\min }$ at lower temperature. In case of $\mathrm{La}_{2 / 3} \mathrm{Ca}_{1 / 3} \mathrm{MnO}_{3}$ the Kondo scattering was found to be negligible for $H \geq 10 \mathrm{kOe}$ [21]. Herein, Kondo scattering had to be taken into account even at $3.0 \mathrm{kOe}$. A good agreement between the experimental data and fitting results provides the possibility to understand resistivity minimum at low temperatures using the theory of both e-e interaction and Kondo-like scattering.

In order to understand the interesting change of the spin scattering strength, dc magnetization measurements in the field cooling (FC) and zero field cooling (ZFC) modes were performed. Figure 6 shows the temperature dependence of ZFC magnetization $M_{\mathrm{ZFC}}$ and FC magnetization $M_{\mathrm{FC}}$ at low field, $H=0.01 \mathrm{~T}$. A sharp PM to FM transition is observed at $T_{\mathrm{C}}$, as determined from the deep in the plot of temperature derivation of $\mathrm{FC}$ magnetization $\left(\mathrm{d} M_{\mathrm{FC}} / \mathrm{d} T\right)$ with $T$. The value of $T_{\mathrm{C}}$ was estimated to be $235 \mathrm{~K}$ which coincides the value of $T_{\mathrm{P}}$ in $\rho(T)$. Large thermo-magnetic irreversibility is observed below $T_{\mathrm{C}}$. The degree of separation of the $M-T$ curves reflects the degree of the strength of the spin disorder. It is well known that this separating phenomenon of MT curve is a possible AFM or the spin-glass (SG) characteristic.

In relation with this observation, the nature of low field MR at selected temperatures has also been studied by varying the magnetic field up to $5.0 \mathrm{kOe}$. The plots of MR are shown in the inset of figure 7, exhibiting different characteristic features at different temperatures. The nature of MR$H$ curves at 200, 175 and $113 \mathrm{~K}$ are similar. In contrast, the MR at $235 \mathrm{~K}\left(T_{\mathrm{C}}\right)$ is linearly depended on field and having the value of MR is $\approx 19 \%$ at $5.0 \mathrm{kOe}$. The MR-H curve at $50 \mathrm{~K}$ in figure 7 shows a sharp decrease initially up to $\approx 15 \mathrm{kOe}$ and then it decreases further almost linearly from $3 \mathrm{kOe}$ to higher field. Initial rapid decrease in MR exhibits the typical feature of grain boundary-assisted tunnelling magnetoresistance while the high-field linear component manifests the intrinsic component attributed to the double-exchange mechanism [15,33]. Here, the contribution of Kondo term decreases monotonically with the increase in field and it is still observed until $3.0 \mathrm{kOe}$. This is reasonable because the contribution to the magneto-resistance from grain boundary effect still exists in the polycrystalline compound as seen in figure 7. If we compare both $M-H$ curve at $10 \mathrm{~K}$ and MR curve at $50 \mathrm{~K}$ as seen in figure 8 , we observe that $M-H$ curve has typical ferromagnetic characteristics where $M$ increases sharply with the increase in field and started to get saturation near 3.0 kOe. MR also shows similar changes correspondingly, i.e., it decreases sharply with the increase in field and then almost linearly at around $3.0 \mathrm{kOe}$. This correlation between electrical transport and magnetic properties at lower

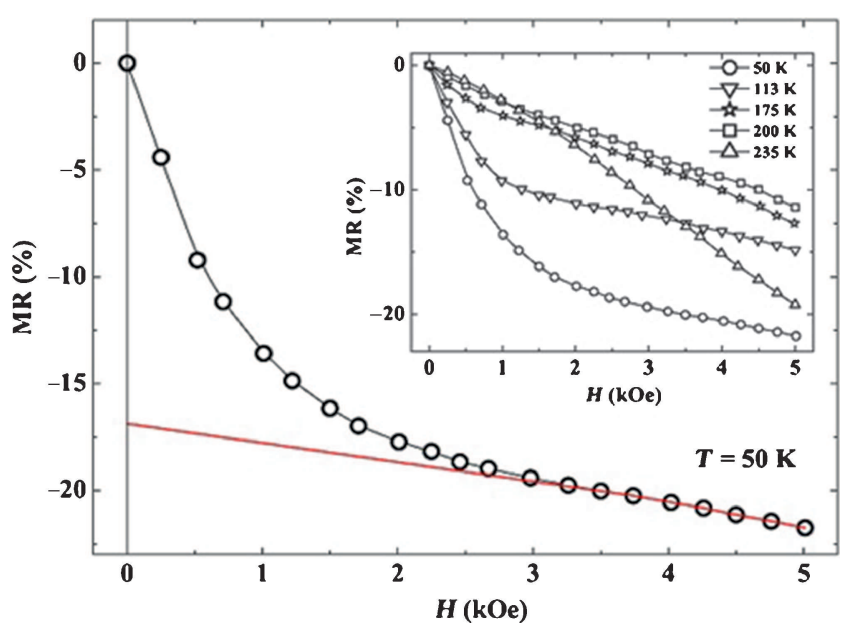

Figure 7. Magnetoresistance (MR) curve is plotted with field $(H)$ at $50 \mathrm{~K}$. High field range satisfying linear dependence is indicated by the straight line. Inset shows the MR at different temperatures. 


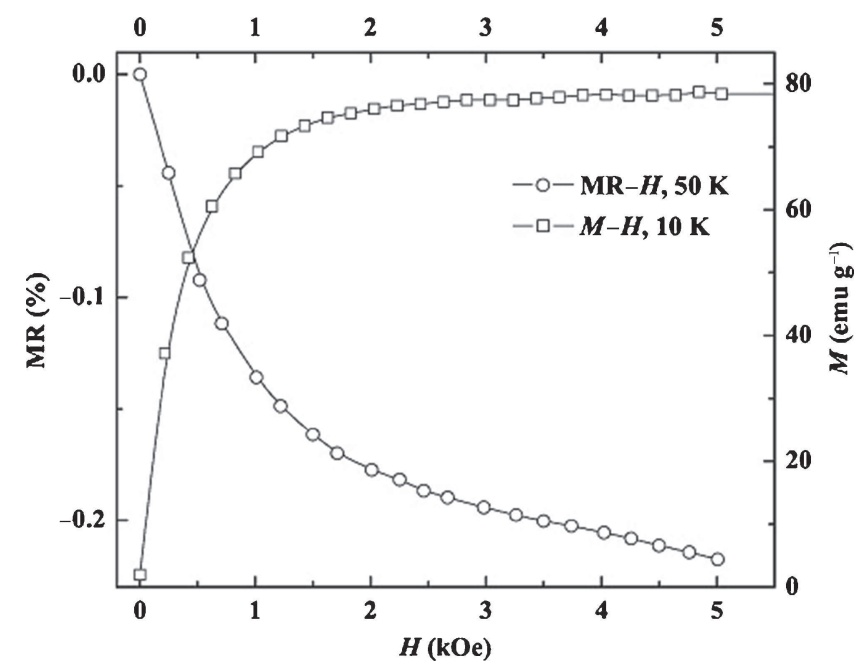

Figure 8. Simultaneous dependence of magnetoresistance (MR) and magnetization $(M)$ on the applied magnetic field at 50 and $10 \mathrm{~K}$, respectively.

field reflects grain boundary contribution in the system at low temperature.

Finally, as a result of $\mathrm{Zn}$ substitution a minor spin-glasslike state was also reported in $\mathrm{LaMn}_{1-x} \mathrm{Zn}_{x} \mathrm{O}_{3}$ [25]. The increase of low-temperature localization in the transport property was attributed to the minor spin-glass-like state in $\mathrm{La}_{09} \mathrm{Mn}_{098} \mathrm{Fe}_{002} \mathrm{O}_{3}$ [22,23] and Y-doped $\left(\mathrm{La}_{1-\delta} \mathrm{Y}_{\delta}\right)_{2 / 3}$ $\mathrm{Ca}_{1 / 3} \mathrm{MnO}_{3}$ [20]. Here, dominant grain boundary effect is considered to be involved with the appearance of minor spinglass-like state due to the $\mathrm{Zn}$ substitution which has an addition localization effect associated with the electron-electron scattering in the transport properties at low temperature.

\section{Conclusions}

In summary, low- $T$ transport properties are investigated in the self-doped compound, $\mathrm{La}_{09} \mathrm{Mn}_{098} \mathrm{Zn}_{002} \mathrm{O}_{3}$. The analysis of the low- $T$ resistivity using phenomenological model indicates the interplay among contributions from electronelectron, Kondo, electron-phonon and electron-magnon scatterings leading to the minimum in the temperature dependence of resistivity. The analysed parameters involved with different scattering processes strongly depend on the applied low magnetic field. The localization effect at low temperature is suggested due to the coexistence of electron-electron and Kondo-like scatterings in the transport properties. The correlation between low-temperature $M$ curve and MR curve supports the grain boundary contribution and grain boundaryassisted tunnelling in the system.

\section{Acknowledgements}

The work was supported by UGC major research project no. 42/908/2013 (SR). We thank Dr S Giri and Dr S Majumdar for accessing their laboratory in Department of Physics,
Indian Association for the Cultivation of Science, India. We would also like to thank Dr B Sarkar, Dr P Roychowdhury, Bijoy Kumar Singha and Sunanda Chatterjee for their consistent inspiration during paper compilation.

\section{References}

[1] Dagotta E, Hotta T and Moreo A 2001 Phys. Rep. 3441

[2] Salamon M B and Jaime M 2001 Rev. Mod. Phys. 73 583

[3] Ramirez A P 1997 J. Phys.: Condens. Matter 98171

[4] Imada M, Fujimori A and Tokura Y 1998 Rev. Mod. Phys. 70 039

[5] Rao C N R and Raveau B 1998 Colossal magnetoresistance, charge ordering and related properties of manganese oxides (Singapore: World Scientific)

[6] Arulraj A, Mahesh R, Subbanna G N, Mahendiran R, Raychaudhuri A K and Rao C N R 1996 J. Solid State Chem. 12787

[7] de Brion S, Ciorcas F, Chaillout G, Lejay P, Radaelli P and Chaillout C 1999 Phys. Rev. B 591304

[8] Troyanchuk I O, Khomchenko V A, Chobot A N and Szymczak H 2004 Phys. Rev. B 69054432

[9] Troyanchuk I O, Khomchenko V A, Chobot A N and Szymczak H 2003 J. Phys.: Condens. Matter 15 6005

[10] Joy P A, Raj Sankar C and Date S K 2002 J. Phys.: Condens. Matter 14 L663

[11] Muroi M, Street R, Cochrane J W and Russell G J 2000 Phys. Rev. B 62 R9268

[12] De K, Majumdar S and Giri S 2007 J. Phys.: Condens. Matter 19096205

[13] De K, Majumdar S and Giri S 2007 J. Phys.: Condens. Matter 19096205

[14] Patra M, De K, Majumdar S and Giri S 2009 Appl. Phys. Lett. 94092506

[15] Patra M, Roy A, De K, Majumdar S and Giri S 2009 Appl. Phys. Lett. 94212107

[16] Rosenberg E, Auslender M, Felner I and Gorodetsky G 2000 J. Appl. Phys. $\mathbf{8 8} 2578$

[17] Auslender M, Karkin A E, Rosenberg E and Gorodetsky G 2001 J. Appl. Phys. 896639

[18] Kumar D, Sankar J, Narayan J, Singh R K and Majumdar A K 2002 Phys. Rev. B 65094407

[19] Rana D S, Markna J H, Parmar R N, Kuberkar D G, Raychaudhuri P, John J and Malik S K 2005 Phys. Rev. B 71 212404

[20] Zhang J, Xu Y, Cao S, Cao G, Zhang Y and Jing C 2005 Phys. Rev. B 72054410

[21] Xu Y, Zhang J, Cao G and Cao S 2006 Phys. Rev. B 73224410

[22] De K, Majumdar S and Giri S 2007 J. Phys. D: Appl. Phys. 405810

[23] De K, Majumdar S and Giri S 2010 J. Magn. Magn. Mater. 322337

[24] Markovich V, Rozenberg E, Gorodetsky G, Mogilyansky D, Revzin B and Pelleg J 2001 J. Appl. Phys. 902347 
[25] Alvarez-Serrano I, Pico C and Veiga M L 2004 Solid State Sci. 61321

[26] Ghosh K, Ogale S B, Ramesh R, Greene R L, Venkatesan T, Gapchup K M, Bathe R and Patil S I 1999 Phys. Rev. B 59533

[27] Seetha Lakshmi L, Dörr K, Nenkov K, Sastry V S and Müller K-H 2007 J. Phys.: Condens. Matter 19236207

[28] El-Fadli Z, Redouane Metni M, Sapina F, Martinez E, Folgado J V and Beltrán A 2002 Chem. Mater. 14688

[29] De K, Ray R, Panda R N, Giri S, Nakamura H and Kohara T 2005 J. Magn. Magn. Mater. 288339

[30] Lee P A and Ramakrishnan T V 1985 Rev. Mod. Phys. 57287
[31] Altshuler B L and Aronov A G 1985 In: Efros A L and Pollak $\mathrm{M}$ eds Electron-electron interactions in disordered systems (Amsterdam: North-Holland)

[32] Tokura Y 1999 Colassal magnetoresistive oxides (New York: Gordon and Breach Science)

[33] Markovich V, Jung G, Fita I, Mogilyansky D, Wu X, Wisniewski A, Puzniak R, Froumin N, Titelman L, Vradman L, Herskowitz M and Gorodetsky G 2008 J. Phys. D: Appl. Phys. 41185001

[34] Schiffer P, Ramiraz A P, Bao W and Cheong S W 1995 Phys. Rev. Lett. 753336 